\title{
An Empirical Measure on the Set of Symbols Occurring in Engineering Mathematics Texts
}

\author{
Stephen M. Watt \\ Ontario Research Centre for Computer Algebra \\ Department of Computer Science \\ University of Western Ontario \\ London Ontario, CANADA N6A 5B7
}

E-mail: watt@orcca.on.ca

\begin{abstract}
Certain forms of mathematical expression are used more often than others in practice. A quantitative understanding of actual usage can provide additional information to improve the accuracy of software for the input of mathematical expressions from scanned documents or handwriting and more natural forms of presentation of mathematical expressions by computer algebra systems. Earlier work has examined this question for the diverse set of articles from the mathematics preprint archive arXiv. org. That analysis showed showed the variance between mathematical areas. The present work analyzes a particular mathematical domain more deeply. We have chosen to examine second year university engineering mathematics as taught in North America as the domain. We have analyzed the set of expressions occurring in the most popular textbooks, weighted by popularity. Assuming that early training influences later mathematical usage, we take this as a model of the set of mathematical expressions used by the population of North American engineers. We present an empirical analysis of the symbols and n-grams occurring in these expressions.
\end{abstract}

\section{Introduction}

This paper concerns computer analysis of mathematical documents. Unlike natural language text, dictionary-based techniques cannot be used easily to guide recognitionthere is no fixed vocabulary of mathematical "words" that may appear in expressions. There is, however, a wellestablished tradition of common mathematical usage: some sub-expressions occur in practice more than others. We wish to use this information to guide the recognition of mathematical text.
In earlier work [1] we have reported on the analysis of some 20,000 articles from the mathematics pre-print server arXiv. org [2]. These articles were classified by area using the MSC subject classification, and we were able to observe that mathematical usage varied considerably by area. We then were able to use the information gathered to construct $n$-grams to improve the recognition accuracy of a pen-based mathematics interface $[3,4]$. The construction of $n$-grams from tree-structured data used a linearization technique to traverse the tree frontier and insert sufficient geometric symbols to keep track of the expression baseline.

We wish to explore further the general approach of using statistical analysis of mathematical corpora to improve the recognition of mathematical expressions by software systems. While our interest is primarily in the area of mathematical handwriting recognition, the same models should be useful in improving the analysis of mathematics in scanned documents by systems such as Infty [5].

One of the difficulties in using the arXiv server is that certain specialized mathematical areas receive an unrepresentative number of articles by particular authors and their idiosyncrasies skew the analysis. For the current work, we have therefore taken an area of general interest that nevertheless exhibits a wide diversity of mathematical notation. We have selected the domain of engineering mathematics as taught in the second year of North American university programs as the scope for the current study.

Second year engineering mathematics is taught as a collection of applied mathematical subjects including such topics as elementary complex analysis and vector calculus. The population that uses these techniques measures in the millions of individuals, so any progress in handling documents with this mathematical content would be useful.

In addition to the use in document analysis and recognition, a statistical study of mathematical expression usage 
can be of interest in other areas. In particular, we would suggest that computer algebra systems could make use of information about what are the preferred forms in practice in order to present their output in the most desirable way.

Earlier work on optical character recognition for typeset mathematical documents touches upon aspects of the current paper. One study [6] considered a collection of 30 English works on pure mathematics and analyzed the scanned images for visual properties of the mathematical characters, such as whether they were touching or abnormal in shape. Another study [7] analyzed a database of 400 document images and noted that expression symbols differed from normal text, that a set of 12 two-dimensional layout structures were used and that the top $150 n$-grams or so were highly representative of the subject categories. The present article does not consider at all the printed appearance of the mathematical text. Instead we take the document source text (ground truth) as given and analyze the symbol and $n$ gram frequences that occur.

The paper is organized as follows: Section 2 presents the problem we study in more detail. Section 3 outlines the methodology we have used to collect and analyze our data. Section 4 presents our first results. Section 5 discusses future work and presents our conclusions.

\section{The Problem}

We are interested in analyzing documents that use engineering mathematics as presented in the second year of the North American university education. Such documents would include engineering documents in professional practice, mathematical textbooks, student assignments and hand-written mathematics by both students and practicing professionals.

While elementary engineering mathematics includes a broad range of activity, the range of mathematical notation used is limited, at least when compared to range of notations for mathematics as a whole. We make the assumption that the notations used in practice will follow to a large those that the practitioners learned while students.

Under this assumption, we are ultimately studying the set of expressions occurring in the collection of textbooks used to teach second year engineering students. We may model the population of expressions used in practice by analyzing the individual textbooks and weighting them by their popularity.

The problem we wish to study is the statistics of the space of mathematical expressions that occur in these texts, with a suitable weighting.

$\begin{array}{clcrr}\text { Rank } & \text { Author } & \text { Reference } & \text { Demand } & \text { Adoptions } \\ 1 & \text { Kreyszig } & {[8]} & 72 \% & 67 \% \\ 2 & \text { Greenberg } & {[9]} & 13 \% & 14 \% \\ 3 & \text { O'Neil } & {[10]} & 7 \% & 8 \% \\ 4 & \text { Jeffrey } & {[11]} & 5 \% & 5 \% \\ 5 & \text { Harman } & {[12]} & 2 \% & 3 \% \\ 6 & \text { Zill } & {[13]} & 1 \% & 1 \% \\ 7 & \text { Potter } & {[14]} & 1 \% & 1 \% \\ 8 & \text { Wylie } & {[15]} & 0 \% & 1 \%\end{array}$

(Source 353 adoptions reported in TDIS.)

\section{Table 1. Second year engineering texts}

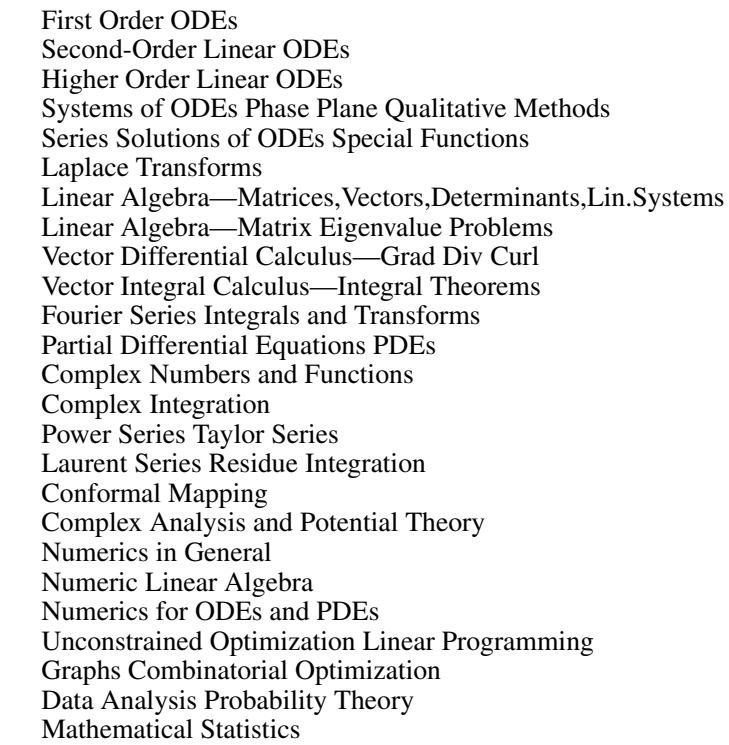

Table 2. Kreyszig table of contents

\section{Methodology}

Corpus Selection The first step in our approach was to identify the most popular textbooks in the area of second year engineering mathematics. Table 1 shows the US college and university bookstore sales for spring for 2006 to fall 2006. From this we see that three titles account for about $90 \%$ of the textbook use. We therefore build our model based on these three titles. The subjects covered in these three texts are shown in Tables 2, 3 and 4.

TEX Sources For each of the three textbooks, we obtained $\mathrm{T}_{\mathrm{E}} \mathrm{X}$ sources for all the mathematical expressions, and then constructed MathML from the $\mathrm{T}_{\mathrm{E}} \mathrm{X}$.

For the texts by Greenberg and O'Neil the author and publisher (respectively) were highly cooperative and provided the $\mathrm{T}_{\mathrm{E}} \mathrm{X}$ sources directly. The sources for the text by O'Neil corresponded to the published version in use today. The sources for the text by Greenberg had somewhat diverged from the published text but not so much as to materially affect the analysis in our opinion. 


\author{
Introduction to Differential Equations \\ Equations of First Order \\ Linear Differential Equations of Second Order and Higher \\ Power Series Solutions \\ Laplace Transform \\ Quantitative Methods-Numerical Solution of DEs \\ Qualitative Methods-Phase Plane and Nonlinear DEs \\ Systems of Linear Algebraic Equations-Gauss Elimination \\ Vector Space \\ Matrices and Linear Equations \\ The Eigenvalue Problem \\ Extension to Complex Case \\ Differential Calculus of Functions of Several Variables \\ Vectors in 3-Space \\ Curves Surfaces and Volumes \\ Scalar and Vector Field Theory \\ Fourier Series Integral Transform \\ Diffusion Equation \\ Wave Equation \\ Laplace Equation \\ Functions of a Complex Variable \\ Conformal Mapping \\ The Complex Integral Calculus \\ Taylor Laurent Series Residue Theorem
}

\section{Table 3. Greenberg table of contents}

\author{
ODEs-First Order Differential Equations \\ ODEs-Second Order Differential Equations \\ ODEs-The Laplace Transform \\ ODEs-Series Solutions \\ ODEs-Numerical Approximation of Solutions \\ Vectors and Linear Algebra-Vectors and Vector Spaces \\ Vectors and Linear Algebra-Matrices and Systems of Linear \\ Equations \\ 8 Vectors and Linear Algebra-Determinants \\ 9 Vectors and Linear Algebra—Eigenvalues Diagonalization and \\ Special Matrices \\ 10 Systems of Linear Differential Equations \\ 11 Qualitative Methods and Systems of Nonlinear Differential \\ Equations \\ Vector Analysis-Vector Differential Calculus \\ Vector Analysis-Vector Integral Calculus \\ Fourier Series \\ The Fourier Integral and Fourier Transforms \\ Fourier Analysis-Special Functions Orthogonal Expansions \\ and Wavelets \\ PDEs-The Wave Equation \\ PDEs-The Heat Equation \\ PDEs-The Potential Equation \\ Geometry and Arithmetic of Complex Numbers \\ Complex Analysis-Complex Functions \\ Complex Analysis-Complex Integration \\ Complex Analysis-Series Representations of Functions \\ Complex Analysis-Singularities and The Residue Theorem \\ Complex Analysis-Conformal Mappings \\ Counting and Probability \\ Statistics
}

\section{Table 4. O'Neil table of contents}

For the text by Kreyszig it was not possible to obtain sources. To obtain the mathematical expressions of the text in electronic form, we first scanned the entire book and used the Infty system to produce $\mathrm{T}_{\mathrm{E}} \mathrm{X}$. In most cases the $\mathrm{T}_{\mathrm{E}} \mathrm{X}$ produced had to be edited by hand to correct errors. This was a highly labour intensive activity that spanned several months. In the end we had a $\mathrm{T}_{\mathrm{E}} \mathrm{X}$ representation for all the mathematical expressions in the text.
MathML Conversion Naive examination of $\mathrm{T}_{\mathrm{E}} \mathrm{X}$ sources does not give the mathematical expressions of a document. This is for two reasons.

The first reason is that typical $\mathrm{TEX}_{\mathrm{E}}$ document markup makes use of a number of macro packages, as well as author-defined macros. These macros have to be expanded to reveal the mathematical expression.

The second reason that the $\mathrm{T}_{\mathrm{E} X} \mathrm{X}$ sources do not give expressions directly is that the $\mathrm{T}_{\mathrm{E}} \mathrm{X}$ representation of mathematics is not grouped as required. For example, most authors would write $\$ a+b \quad c \$$ rather than $\$ a+\{b \quad c\} \$$. While it is true that a coarsening of the $\mathrm{T}_{\mathrm{E} X}$ layout tree would correspond to a coarsening of the mathematical expression tree, it is still in general necessary to regroup the $\mathrm{T}_{\mathrm{E}} \mathrm{X}$ representation.

We used our TEX to MathML [16] converter[17], described elsewhere [18], to resolve these difficulties, and performed our analysis on the resulting MathML expressions. The benefit of this approach was that the expressions treated were (for the most part) complete, well formed, and grouped appropriately. The difficulty with the approach was that not all the complexities of $\mathrm{T}_{\mathrm{E}} \mathrm{X}$ were handled, and a small number of expressions were incorrectly translated. However, since we are interested in the most frequently occurring expressions, the incomplete handling of infrequently occurring expressions is not, in principle, a problem. The conversion process has been described in more detail elsewhere [1].

Analysis We grouped the chapters of each text into the general categories shown in Table 5 and analyzed the mathematical expressions for each subject/author combination, for each author with subjects combined (as given in the text), and for each subject with authors combined by weight.

In each case, we computed the individual symbol frequencies (normalized to total 1 ) and $n$-gram frequencies for $n=2,3,4,5$. To compute the $n$-grams, we converted the expressions to strings by traversing the frontier of the expression trees in writing order. The resulting strings were over the alphabet of leaf symbols extended by $\langle$ sub $\rangle$, $\langle/$ sub $\rangle$, $\langle$ sup $\rangle$, $\langle/$ sup $\rangle,\langle$ frac $/\rangle$ and $<$ root $/>$. These symbols captured transitions from the expression baseline to subscripts and superscripts as well as built up fractions and radicals. The $n$-grams were then tallied using sliding windows over these strings.

\section{Results}

Single Symbols Table 6 shows the frequencies of the most commonly occurring symbols in the entire set of expressions. These are presented with the absolute symbol count for each author and as a percentage of all symbols, weighted by author. The relative weights used were 
- Ordinary Differential Equations

(Kreyszig 1-6, Greenberg 1-7, O’Neil 1-5 \& 10-11)

- Linear Algebra

(Kreyszig 7-8, Greenberg 8-11 \& 14, O’Neil 6-9)

- Vector Calculus

(Kreyszig 9-10, Greenberg 16, O’Neil 12-13)

- Partial Differential Equations

(Kreyszig 12, Greenberg 18-20, O’Neil 17-19

- Fourier Analysis

(Kreyszig 11, Greenberg 17, O’Neil 14-16)

- Multivariable Calculus (Greenberg 13\&15)

- Complex Analysis (Kreyszig 13-18, Greenberg 12\&21-24, O’Neil 20-25)

- Numerical Analysis (Kreyszig 19-21)

- Linear Programming (Kreyszig 22)

- Graph Theory (Kreyszig 23)

- Probability and Statistics (Kreyszig 24-25, O’Neil 26-27)

Table 5. Subject Groupings

$72:: 13:: 7$. We see that the most popular symbols were common among all the authors, although the rank of the symbols varied somewhat from author to author. The total number of mathematical symbols occurring in the texts were (368 267 and 467044 and 391 602, respectively).

Tables 7 and 8 show the most commonly occurring symbols for the second year engineering versions of complex analysis and partial differential equations, respectively. We see that the curve of declining relative frequency of the most popular symbols is similar between the areas, with a few outlying points (such as $z$ being very popular for complex analysis). This same pattern was observed for all subject areas.

The cumulative frequency of symbols is shown in Figure 1 with one curve for each subject and one for the weighted combination. Figure 2 shows the same curves on a log plot, from which it is possible to see that the symbols follow an exponential distribution. Tables 9 and 10 show the most popular 2-grams and 5-grams respectively for the three authors of the selected corpus as well as from two comparison texts $[19,20]$. The $n$-grams have a qualitatively similar declining frequency pattern as the symbols, but this time in a much larger space.

The total number of $n$-grams (for any $n$ ) was 479388 for Kreyzig, 562297 for Greenberg and 477268 for O'Neil. The total number of different bigrams was 5992 (Kreyszig),

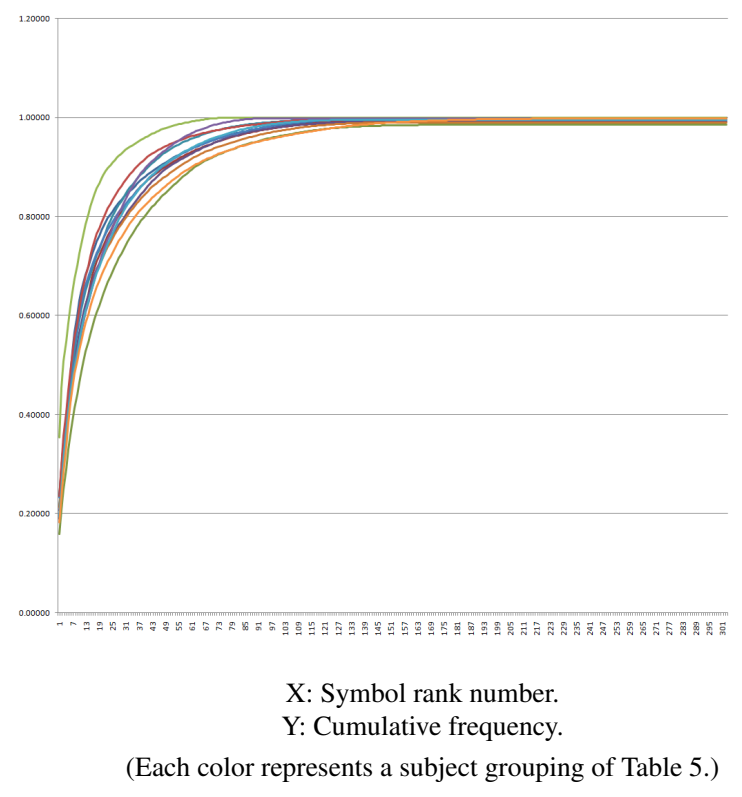

Figure 1. Cumulative symbol freq. by subject

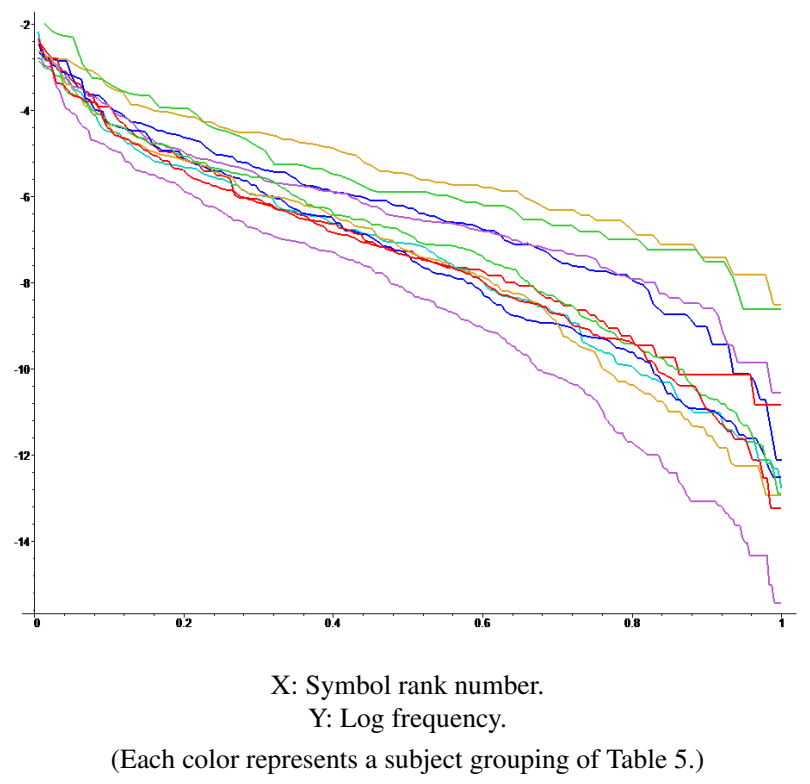

Figure 2. Log frequencies

7056 (Greenberg) and (5 442) O'Neil. The total number of different 5-grams was 140306 (Kreyszig), 146507 (Greenberg), 126232 (O’Neil).

Figure 3 shows the cumulative frequency for all distinct $n$-grams occurring in the text by Kreyszig. The highest curve is for $n=2$ and they are in order to the lowest curve for $n=5$. We find it remarkable that even though the rank- 


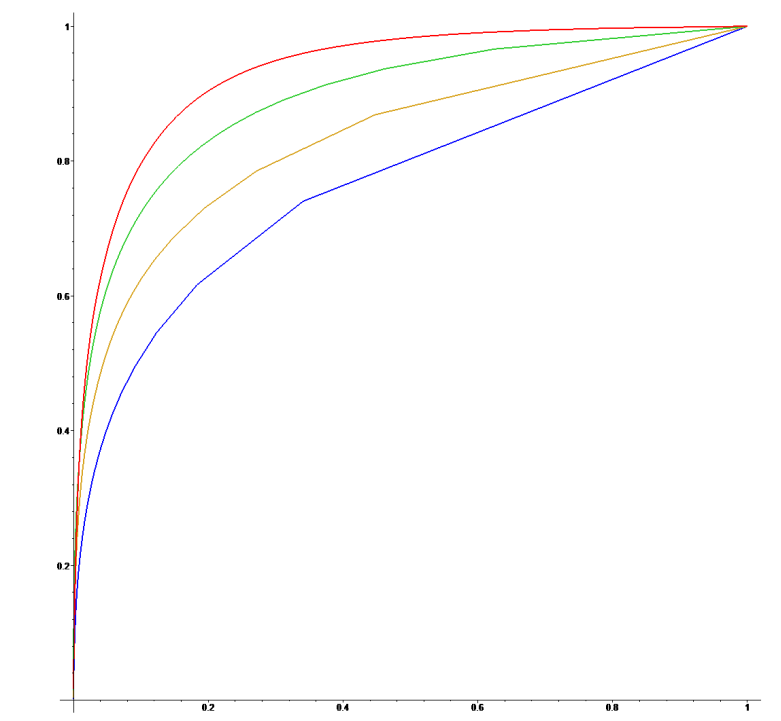

From top to bottom, curves count 2-, 3-, 4- and 5-grams for entire corpus.

Figure 3. $n$-gram cumulative frequency

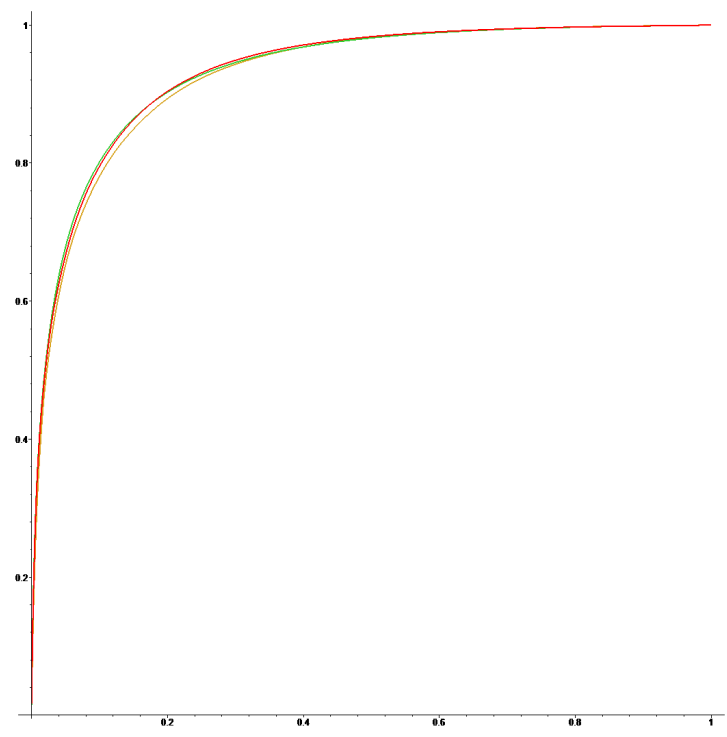

Figure 4. Bigram cumul. frequency per author

ing of the particular $n$-grams is different for the each author, the cumulative $n$-gram frequency curves are virtually identical for each author. Figure 4 shows the cumulative frequency of bigrams, ordered by popularity, for the three authors.

\section{Conclusions}

Earlier work had shown that statistical analysis of mathematical research documents could produce $n$-grams that improve mathematical handwriting recognition rates for highlevel mathematics.

We have have therefore been motivated to define a more elementary corpus of mathematics that would be more widely applicable and analyze its statistical structure. We have selected second year engineering mathematics as taught in North America as the subject and have analyzed the expressions that occur in the textbooks are adopted in more than $90 \%$ of the classes. We are then able to produce statistics weighted by the popularity of the textbooks, thus modeling the set of expressions that are used in practice.

Analyzing the population of symbols and $n$-grams that occur in these texts, we are able to determine the most popular symbols and $n$-grams by subject. The exponential drop in number of occurrences from the highest ranked symbols and $n$-grams to the lowest, means that a compact database can contain all of the frequently occurring items. Thus applications, even those for portable devices, could use these statistics to guide their recognition.

Future work will explore how well this performs in practice for elementary engineering mathematics.

\section{Acknowledgments}

The author would like to thank Michael Greenberg, Peter O'Neil, Prentice-Hall and Thomas-Nelson for the use of their materials. We also thank Robert Lopez and Maplesoft for the use of additional materials. We thank Jeliazko Polihronov for his assistance in gathering the data and Elena Smirnova for her work on the $n$-gram analysis software. This work was supported in part by grants from the Natural Sciences and Engineering Research Council of Canada, Microsoft and Maplesoft.

\section{References}

[1] Clare M. So and Stephen M. Watt, Determining Empirical Properties of Mathematical Expression Use, pp. 361375, Proc. Fourth International Conference on Mathematical Knowledge Management, (MKM 2005), July 15-17 2005, Bremen Germany, Springer Verlag LNCS 3863.

[2] ArXiv e-Print Archive. http: //arxiv.org

[3] Elena Smirnova and Stephen M. Watt, Combining Prediction and Recognition to Improve On-Line Mathematical Character Recognition (submitted). Available as ORCCA Technical Report TR-06-06. http://www. orcca.on . ca/TechReports/2006/TR-06-06.html

[4] Elena Smirnova and Stephen M. Watt, A pen-based mathematical environment "Mathink" (submitted). Available as ORCCA Technical Report TR-06-05. http://www.orcca.on.ca/TechReports / 2006/TR-06-05.html 
[5] M.Suzuki, F.Tamari, R.Fukuda, S.Uchida, T.Kanahori, Infty - an integrated OCR system for mathematical documents, Proceedings of ACM Symposium on Document Engineering 2003, Grenoble, Ed. C.Vanoirbeek, C.Roisin, E. Munson, 2003, pp.95-104

[6] S.Uchida, A.Nomura, and M.Suzuki, Quantitative analysis of mathematical documents, International Journal on Document Analysis and Recognition, Vol.7, Issue.4, pp.211-218. (September 2005)

[7] U.Garain and B.B Chaudhuri, A corpus for OCR research on mathematical expressions, International Journal on Document Analysis and Recognition, Vol.7, Issue.4, pp.241-259. (September 2005)

[8] Erwin Kreyszig, Advanced Engineering Mathematics, $8^{\text {th }}$ edition, John Wiley \& Sons 1999.

[9] Michael Greenberg, Advanced Engineering Mathematics, $2^{\text {nd }}$ edition, Prentice Hall 1998.

[10] Peter O'Neil, Advanced Engineering Mathematics, $5^{\text {th }}$ edition, Thomson-Nelson 2003.

[11] Alan Jeffrey, Advanced Engineering Mathematics, $2^{\text {nd }}$ edition, Academic Press 2002.

[12] Thomas L. Harman, James Dabney, Norman J. Richert, Advanced Engineering Mathematics with MATLAB, $2^{\text {nd }}$ edition, Thomson-Engineering 2000.

[13] Dennis G. Zill, Michale R. Cullen, Advanced Engineering Mathematics, $3^{\text {rd }}$ edition, Jones and Bartlett 2006.

[14] Merle C. Potter, Advanced Engineering Mathematics, $3^{\text {th }}$ edition, Oxford University Press 2005.

[15] C. Ray Wylie, Advanced Engineering Mathematics, $6^{\text {th }}$ edition, McGraw-Hill 1995.

[16] David Carlisle, Patrick Ion, Robert Miner, Nico Poppelier, Editors. Mathematical Markup Language (MathML) Version 2.0 (Second Edition). W3C Recommendation. http://www.w3.org/TR/2003/ REC-MathML2-20031021/. October 21, 2003.

[17] Ontario Research Centre for Computer Algebra. On-line TeX to MathML translator. http://www.orcca.on. $\mathrm{ca} /$ MathML/texmml/textomml.html

[18] Stephen M. Watt. Implicit Mathematical Semantics in Conversion between $\mathrm{T}_{\mathrm{E}} \mathrm{X}$ and MathML, TUGBoat, Vol 23, No 1 (2002)

[19] R. Lopez, Advanced Engineering Mathematics wtih Maple, Maplesoft 2005.

[20] J. Weiner, The Mathematics Survival Kit, Maplesoft 2006.

\begin{tabular}{|c|c|c|c|c|}
\hline \multirow[t]{2}{*}{ Symbol } & \multirow{2}{*}{$\begin{array}{l}\text { Weighted } \\
\text { Freq. (\%) }\end{array}$} & \multicolumn{3}{|c|}{ Symbol Counts } \\
\hline & & Kreyszig & Greenberg & O'Neil \\
\hline 1 & 6.16415 & 24519 & 23209 & 20345 \\
\hline 2 & 6.15918 & 24436 & 22613 & 21886 \\
\hline$=$ & 5.89883 & 22906 & 26202 & 19275 \\
\hline 0 & 5.13055 & 20436 & 19623 & 16164 \\
\hline( & 5.08432 & 18162 & 26262 & 27777 \\
\hline ) & 5.08387 & 18158 & 26257 & 27804 \\
\hline$x$ & 4.97402 & 18271 & 28243 & 17918 \\
\hline- & 3.82436 & 14609 & 15625 & 17152 \\
\hline+ & 3.12976 & 11906 & 14648 & 11711 \\
\hline$y$ & 2.94812 & 11400 & 13191 & 9996 \\
\hline 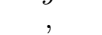 & 2.53506 & 9796 & 12571 & 6784 \\
\hline$n$ & 2.11526 & 8016 & 9681 & 8577 \\
\hline$z$ & 1.88590 & 7447 & 7238 & 6593 \\
\hline 3 & 1.87252 & 7225 & 7603 & 7706 \\
\hline 믐 & 1.73059 & 6386 & 7715 & 9163 \\
\hline$t$ & 1.71003 & 5771 & 9800 & 11446 \\
\hline & 1.62134 & 6234 & 4510 & 10083 \\
\hline 4 & 1.42027 & 5694 & 4119 & 6097 \\
\hline$f$ & 1.30925 & 4926 & 6522 & 4874 \\
\hline & 1.24019 & 4427 & 7757 & 4749 \\
\hline$a$ & 1.21198 & 4627 & 6305 & 3390 \\
\hline 5 & 1.14952 & 4771 & 3030 & 3674 \\
\hline$i$ & 0.91795 & 3451 & 4251 & 3940 \\
\hline$u$ & 0.91478 & 3392 & 5740 & 2121 \\
\hline$c$ & 0.89854 & 3638 & 3096 & 2727 \\
\hline$s$ & 0.87843 & 3539 & 3742 & 1756 \\
\hline$d$ & 0.84576 & 2761 & 6929 & 3460 \\
\hline$e$ & 0.84518 & 3010 & 4819 & 4019 \\
\hline | & 0.81767 & 3270 & 2962 & 2691 \\
\hline$\pi$ & 0.76648 & 2913 & 2710 & 4243 \\
\hline / & 0.75086 & 2849 & 3730 & 2557 \\
\hline 6 & 0.72635 & 2981 & 1648 & 3088 \\
\hline$k$ & 0.71945 & 2892 & 2217 & 2807 \\
\hline & 0.70123 & 2698 & 3114 & 2558 \\
\hline L & 0.70104 & 2697 & 3110 & 2565 \\
\hline$m$ & 0.64372 & 2712 & 2033 & 1114 \\
\hline 8 & 0.55862 & 2374 & 963 & 1977 \\
\hline$r$ & 0.55619 & 2008 & 3348 & 2085 \\
\hline$b$ & 0.54474 & 2080 & 2731 & 1678 \\
\hline 9 & 0.49895 & 2144 & 741 & 1698 \\
\hline $\sin$ & 0.46307 & 1704 & 2190 & 2310 \\
\hline$v$ & 0.45379 & 1679 & 2863 & 1067 \\
\hline$j$ & 0.44919 & 1783 & 1565 & 1716 \\
\hline 7 & 0.44045 & 1818 & 890 & 1930 \\
\hline$\ldots$ & 0.43961 & 1918 & 957 & 723 \\
\hline $\cos$ & 0.42582 & 1667 & 1409 & 1988 \\
\hline$\partial$ & 0.41059 & 1363 & 2621 & 2578 \\
\hline$C$ & 0.40904 & 1470 & 2918 & 906 \\
\hline A & 0.40878 & 1517 & 2092 & 1660 \\
\hline$<$ & 0.39223 & 1334 & 2919 & 1487 \\
\hline$p$ & 0.38261 & 1249 & 2972 & 1815 \\
\hline$\leq$ & 0.38081 & 1534 & 1016 & 1693 \\
\hline $\int$ & 0.37320 & 1216 & 2585 & 2276 \\
\hline$w$ & 0.35636 & 1505 & 985 & 793 \\
\hline$\infty$ & 0.34904 & 1093 & 2796 & 2019 \\
\hline$A$ & 0.34528 & 1294 & 1989 & 927 \\
\hline$F$ & 0.34459 & 1396 & 1402 & 708 \\
\hline$L$ & 0.32925 & 1097 & 2217 & 1848 \\
\hline$\lambda$ & 0.31876 & 1210 & 1817 & 722 \\
\hline$h$ & 0.29846 & 1195 & 1176 & 822 \\
\hline$\theta$ & 0.27871 & 926 & 2266 & 995 \\
\hline$T$ & 0.27346 & 1078 & 1282 & 619 \\
\hline$R$ & 0.26417 & 1033 & 1114 & 878 \\
\hline$P$ & 0.26299 & 1021 & 1041 & 1057 \\
\hline$D$ & 0.24927 & 780 & 2531 & 629 \\
\hline
\end{tabular}

(Top 65 out of 305)

Table 6. Top Symbols: All subjects combined 


\begin{tabular}{|c|c|c|c|c|}
\hline \multirow[t]{2}{*}{ Symbol } & \multirow{2}{*}{$\begin{array}{l}\text { Weighted } \\
\text { Freq. (\%) }\end{array}$} & \multicolumn{3}{|c|}{ Symbol Counts } \\
\hline & & Kreyszig & Greenberg & O'Neil \\
\hline$z$ & 11.28007 & 5740 & 4155 & 4670 \\
\hline$=$ & 6.19577 & 3052 & 2879 & 2566 \\
\hline$)$ & 5.76133 & 2664 & 2761 & 4145 \\
\hline( & 5.75744 & 2661 & 2761 & 4152 \\
\hline 1 & 5.59297 & 2790 & 2559 & 2006 \\
\hline 2 & 5.21226 & 2520 & 2669 & 2223 \\
\hline- & 4.02399 & 1912 & 2075 & 2058 \\
\hline 0 & 3.88584 & 1934 & 1609 & 1756 \\
\hline+ & 3.71409 & 1793 & 1845 & 1719 \\
\hline$i$ & 2.95919 & 1358 & 1609 & 1888 \\
\hline$n$ & 2.94910 & 1504 & 1016 & 1315 \\
\hline | & 2.78406 & 1381 & 1120 & 1282 \\
\hline$x$ & 2.45995 & 1125 & 1621 & 1086 \\
\hline$f$ & 1.98821 & 926 & 1004 & 1262 \\
\hline , & 1.69837 & 839 & 842 & 579 \\
\hline y & 1.60176 & 759 & 903 & 699 \\
\hline$\pi$ & 1.30730 & 631 & 537 & 815 \\
\hline$C$ & 1.18192 & 570 & 855 & 56 \\
\hline 3 & 1.13346 & 527 & 683 & 524 \\
\hline$d$ & 1.10683 & 477 & 824 & 634 \\
\hline / & 1.09869 & 519 & 619 & 498 \\
\hline$e$ & 1.08463 & 507 & 585 & 599 \\
\hline$a$ & 0.95106 & 383 & 893 & 497 \\
\hline 4 & 0.85898 & 421 & 382 & 411 \\
\hline$w$ & 0.84605 & 406 & 344 & 560 \\
\hline$\infty$ & 0.75755 & 352 & 459 & 348 \\
\hline$u$ & 0.72164 & 331 & 476 & 307 \\
\hline$<$ & 0.68370 & 309 & 511 & 230 \\
\hline$s$ & 0.63924 & 338 & 245 & 107 \\
\hline$t$ & 0.62265 & 248 & 397 & 704 \\
\hline$\theta$ & 0.56642 & 273 & 255 & 316 \\
\hline$r$ & 0.55408 & 281 & 190 & 266 \\
\hline$\leq$ & 0.54975 & 279 & 134 & 363 \\
\hline $\bar{z}$ & 0.52955 & 308 & 39 & 85 \\
\hline$R$ & 0.52740 & 261 & 285 & 131 \\
\hline$c$ & 0.51782 & 226 & 369 & 296 \\
\hline$D$ & 0.51152 & 186 & 572 & 317 \\
\hline $\cos$ & 0.50425 & 247 & 201 & 286 \\
\hline S & 0.49176 & 154 & 350 & 949 \\
\hline $\int$ & 0.48189 & 210 & 324 & 315 \\
\hline$\ldots$ & 0.48000 & 263 & 141 & 59 \\
\hline $\sin$ & 0.47708 & 220 & 235 & 338 \\
\hline$v$ & 0.44584 & 178 & 438 & 214 \\
\hline$b$ & 0.42329 & 196 & 215 & 279 \\
\hline 1 & 0.40738 & 162 & 379 & 242 \\
\hline$F$ & 0.39388 & 220 & 90 & 50 \\
\hline$m$ & 0.38962 & 205 & 100 & 168 \\
\hline$\sum$ & 0.37173 & 183 & 140 & 216 \\
\hline 5 & 0.37171 & 179 & 204 & 141 \\
\hline$\Phi$ & 0.36030 & 215 & 5 & 38 \\
\hline$\Delta$ & 0.34065 & 176 & 183 & 5 \\
\hline$\rightarrow$ & 0.32410 & 135 & 230 & 258 \\
\hline$\oint$ & 0.32389 & 149 & 191 & 175 \\
\hline$\square$ & 0.29125 & 156 & - & 234 \\
\hline 6 & 0.28148 & 139 & 93 & 183 \\
\hline$k$ & 0.26354 & 122 & 122 & 196 \\
\hline$L$ & 0.25968 & 132 & 93 & 114 \\
\hline$\square$ & 0.25574 & 108 & 207 & 140 \\
\hline & 0.25324 & 100 & 247 & 137 \\
\hline & 0.25234 & 100 & 247 & 131 \\
\hline$\partial$ & 0.24631 & 106 & 145 & 214 \\
\hline$>$ & 0.24236 & 126 & 79 & 90 \\
\hline$!$ & 0.20472 & 101 & 100 & 74 \\
\hline$\not \neq$ & 0.19061 & 92 & 82 & 112 \\
\hline$\epsilon$ & 0.18347 & 98 & 81 & - \\
\hline $\ln$ & 0.18341 & 100 & 42 & 50 \\
\hline
\end{tabular}

\begin{tabular}{|c|c|c|c|c|}
\hline \multirow[t]{2}{*}{ Symbol } & \multirow{2}{*}{$\begin{array}{l}\text { Weighted } \\
\text { Freq. (\%) }\end{array}$} & \multicolumn{3}{|c|}{ Symbol Counts } \\
\hline & & Kreyszig & Greenberg & O'Neil \\
\hline$=$ & 7.22187 & 1362 & 3661 & 2625 \\
\hline$x$ & 7.04832 & 1289 & 4080 & 2874 \\
\hline( & 6.44756 & 1125 & 3923 & 3745 \\
\hline$)$ & 6.43967 & 1123 & 3922 & 3751 \\
\hline 2 & 5.54914 & 1064 & 2378 & 2187 \\
\hline 0 & 4.82981 & 827 & 3394 & 2562 \\
\hline$u$ & 4.28608 & 822 & 2355 & 949 \\
\hline 1 & 3.35806 & 607 & 2117 & 1306 \\
\hline$n$ & 3.33931 & 659 & 1175 & 1200 \\
\hline$t$ & 3.10607 & 594 & 2205 & 1859 \\
\hline$y$ & 2.63211 & 480 & 1442 & 1224 \\
\hline- & 2.38819 & 419 & 1485 & 1283 \\
\hline+ & 2.02753 & 354 & 1521 & 764 \\
\hline , & 2.00038 & 308 & 2050 & 1031 \\
\hline$c$ & 1.68841 & 334 & 645 & 514 \\
\hline$r$ & 1.67920 & 325 & 817 & 446 \\
\hline$\pi$ & 1.66333 & 317 & 593 & 875 \\
\hline$f$ & 1.38602 & 260 & 722 & 512 \\
\hline$\partial$ & 1.32067 & 245 & 285 & 1130 \\
\hline$m$ & 1.13741 & 249 & 165 & 114 \\
\hline$L$ & 1.08369 & 184 & 748 & 634 \\
\hline$w$ & 0.97867 & 217 & 153 & 12 \\
\hline $\sin$ & 0.97415 & 184 & 412 & 463 \\
\hline . & 0.93572 & 136 & 649 & 1129 \\
\hline$d$ & 0.88630 & 171 & 301 & 434 \\
\hline / & 0.85220 & 165 & 418 & 220 \\
\hline$s$ & 0.83542 & 164 & 457 & 91 \\
\hline$F$ & 0.79309 & 161 & 277 & 173 \\
\hline$\infty$ & 0.73690 & 117 & 589 & 525 \\
\hline$\theta$ & 0.70563 & 130 & 396 & 281 \\
\hline 4 & 0.69761 & 132 & 318 & 293 \\
\hline 3 & 0.66666 & 120 & 419 & 274 \\
\hline$G$ & 0.65305 & 145 & 83 & 30 \\
\hline$B$ & 0.63865 & 138 & 143 & 39 \\
\hline$k$ & 0.63278 & 109 & 420 & 354 \\
\hline 5 & 0.61589 & 110 & 445 & 193 \\
\hline$p$ & 0.60337 & 130 & 127 & 58 \\
\hline$e$ & 0.58116 & 100 & 423 & 275 \\
\hline$a$ & 0.57791 & 102 & 441 & 179 \\
\hline$\varphi$ & 0.55596 & 120 & 93 & 82 \\
\hline $\cos$ & 0.55098 & 108 & 112 & 333 \\
\hline$A$ & 0.53950 & 106 & 244 & 129 \\
\hline ' & 0.51395 & 74 & 546 & 369 \\
\hline $\int$ & 0.50346 & 92 & 164 & 388 \\
\hline$\alpha$ & 0.48979 & 82 & 513 & 68 \\
\hline$R$ & 0.43566 & 86 & 130 & 189 \\
\hline$v$ & 0.43559 & 92 & 144 & 15 \\
\hline$\lambda$ & 0.41367 & 88 & 41 & 134 \\
\hline$<$ & 0.40432 & 73 & 950 & 470 \\
\hline$i$ & 0.39696 & 77 & 227 & 53 \\
\hline$b$ & 0.38346 & 61 & 375 & 173 \\
\hline$T$ & 0.32036 & 54 & 163 & 280 \\
\hline 8 & 0.29625 & 61 & 63 & 100 \\
\hline$\Delta$ & 0.28748 & 48 & 259 & 103 \\
\hline$\sum$ & 0.26555 & 49 & 127 & 135 \\
\hline$z$ & 0.26496 & 39 & 248 & 216 \\
\hline$\ldots$ & 0.25696 & 56 & 60 & 12 \\
\hline 1 & 0.25482 & 36 & 231 & 257 \\
\hline & 0.25441 & 36 & 230 & 256 \\
\hline$\stackrel{\stackrel{L}{C}}{ }$ & 0.24920 & 49 & 135 & 27 \\
\hline$*$ & 0.24767 & 55 & 33 & 9 \\
\hline$\omega$ & 0.24534 & 16 & 172 & 797 \\
\hline$g$ & 0.24349 & 41 & 164 & 157 \\
\hline$\leq$ & 0.24063 & 36 & 69 & 403 \\
\hline $\bar{J}$ & 0.23427 & 44 & 132 & 71 \\
\hline$W$ & 0.22762 & 51 & 28 & - \\
\hline
\end{tabular}

(Top 65 out of 194.)

(Top 65 out of 193.)

Table 7. Top Symbols: Complex Analysis

Table 8. Top Symbols: PDEs 


\begin{tabular}{|c|c|c|c|c|c|c|c|c|c|}
\hline \multicolumn{2}{|c|}{ Kreyszig } & \multicolumn{2}{|c|}{ Greenberg } & \multicolumn{2}{|c|}{ O'Neil } & \multicolumn{2}{|c|}{ Lopez [19] } & \multicolumn{2}{|c|}{ MSKit [20] } \\
\hline Freq $(\%)$ & Sequence & Freq $(\%)$ & Sequence & Freq $(\%)$ & Sequence & Freq $(\%)$ & Sequence & Freq $(\%)$ & Sequence \\
\hline 0.015609 & $1^{\langle/ \mathrm{sub}\rangle}$ & 0.013729 & $(x$ & 0.013652 & $=$ & 0.015275 & $\langle\sup \rangle 2$ & 0.026046 & $(x$ \\
\hline 0.013716 & $\langle$ sub $\rangle 1$ & 0.012302 & $1\langle/$ sub $\rangle$ & 0.013640 & $\langle\sup \rangle 2$ & 0.015171 & $2\langle/$ sup $\rangle$ & 0.019772 & $x)$ \\
\hline 0.012866 & $2\langle/$ sup $\rangle$ & 0.011704 & $2\langle/$ sup $\rangle$ & 0.013500 & $2\langle/$ sup $\rangle$ & 0.012549 & $(x$ & 0.018647 & $2\langle/$ sup $\rangle$ \\
\hline 0.012828 & $\langle\sup \rangle \mid 2$ & 0.011643 & $\langle$ sup $\rangle 2$ & 0.012630 & $(x$ & 0.009457 & )$=$ & 0.017966 & $\langle\sup \rangle \mid 2$ \\
\hline 0.011231 & $2\langle/$ sub $\rangle$ & 0.011210 & )$=$ & 0.008977 & $t)$ & 0.009434 & 00 & 0.015542 & $x^{\langle\sup \rangle}$ \\
\hline 0.011127 & $\langle$ sub $\rangle$ & 0.010881 & $\langle$ sub $\rangle 1$ & 0.008486 & $x)$ & 0.009044 & -1 & 0.013704 & )$=$ \\
\hline 0.009607 & )$=$ & 0.008806 & $=0$ & 0.008406 & $1^{\langle/ \mathrm{sub}\rangle}$ & 0.008534 & $x)^{-}$ & 0.010710 & $x+$ \\
\hline 0.009482 & $(x$ & 0.008434 & $x)$ & 0.008301 & -1 & 0.007400 & $1\langle$ frac $/\rangle$ & 0.010583 & $n($ \\
\hline 0.009255 & $x^{\langle\mathrm{sub}\rangle}$ & 0.007672 & $2^{\langle/ \text {sub }\rangle}$ & 0.007969 & $e^{\langle\text {sup }\rangle}$ & 0.007261 & $t)$ & 0.009933 & -1 \\
\hline 0.008517 & $\langle/$ sub $\rangle=$ & 0.007556 & $e^{\langle\sup \rangle}$ & 0.007835 & $0\langle/ \mathrm{sub}\rangle$ & 0.007216 & $1\langle/$ sub $\rangle$ & 0.009696 & $x-$ \\
\hline 0.007745 & $0\langle/$ sub $\rangle$ & 0.007504 & $\langle$ sub $\rangle 2$ & 0.007278 & $(t$ & 0.006365 & $\langle/ \sup \rangle+$ & 0.008967 & $\langle/ \sup \rangle+$ \\
\hline 0.007711 & $=0$ & 0.006287 & $0\langle/$ sub $\rangle$ & 0.007161 & $\langle/$ sub $\rangle\langle$ sup $\rangle$ & 0.005821 & $\langle$ sub $\rangle 1$ & 0.008650 & $y=$ \\
\hline 0.007060 & $\langle$ sub $\rangle 0$ & 0.006233 & $x^{\langle\mathrm{sub}\rangle}$ & 0.006839 & $\langle$ sub $\rangle 0$ & 0.005767 & $0:=$ & 0.008618 & $x=$ \\
\hline 0.007030 & $y^{\langle\text {sub }\rangle}$ & 0.006224 & $\langle/$ sub $\rangle=$ & 0.006631 & $\langle$ sub $\rangle$ & 0.005740 & $(t$ & 0.008365 & $\langle$ root $/\rangle 2$ \\
\hline 0.006699 & -1 & 0.006182 & $\langle$ sub $\rangle n$ & 0.006592 & $1\langle$ frac $/\rangle$ & 0.005608 & $x^{\langle\sup \rangle}$ & 0.008349 & $1\langle$ frac $/\rangle$ \\
\hline 0.006676 & $=1$ & 0.006182 & $\langle\sup \rangle^{\wedge}$ & 0.006258 & $=0$ & 0.005419 & $e^{\langle\text {sup }\rangle}$ & 0.008333 & $d x$ \\
\hline 0.006362 & 0 . & 0.006182 & $\wedge /$ sup $\rangle$ & 0.006033 & $\langle$ sub $\rangle 1$ & 0.005344 & $<\operatorname{root} />2$ & 0.007002 & +1 \\
\hline 0.006349 & $\langle/$ sub $\rangle\langle$ sup $\rangle$ & 0.006015 & -1 & 0.005804 & $\langle/ \sup \rangle+$ & 0.005287 & $\langle$ sub $\rangle k$ & 0.006939 & $2 x$ \\
\hline 0.006187 & $\langle\mathrm{sub}\rangle n$ & 0.005707 & $\langle$ sub $\rangle 0$ & 0.005798 & $f($ & 0.005178 & $\langle$ frac $/\rangle 2$ & 0.006876 & $f($ \\
\hline 0.005891 & $x)$ & 0.005471 & $\langle/$ sub $\rangle\langle$ sup $\rangle$ & 0.005506 & $=1$ & 0.005061 & $f($ & 0.006464 & $3\langle/$ sup $\rangle$ \\
\hline 0.005847 & $\langle$ sup $\rangle$, & 0.005457 & $\langle/$ sub $\rangle$ & 0.005479 & $x\langle\sup \rangle$ & 0.005007 & 10 & 0.006432 & $=1$ \\
\hline 0.005831 & $\lambda\langle/ \sup \rangle$ & 0.005333 & $t)$ & 0.005372 & $n($ & 0.004949 & $2\langle/$ sub $\rangle$ & 0.005877 & $\langle/ \sup \rangle-$ \\
\hline 0.005706 & $e^{\langle\text {sup }\rangle}$ & 0.004978 & $n\langle/$ sub $\rangle$ & 0.005139 & $\langle/ \sup \rangle$ & 0.004941 & $=1$ & 0.005656 & $\langle/$ sub $\rangle$ \\
\hline 0.005546 & $\langle/ \sup \rangle+$ & 0.004962 & in & 0.005133 & $2^{\langle/ \text {sub }\rangle}$ & 0.004806 & $\langle$ sub $\rangle 2$ & 0.005640 & )$\langle\sup \rangle$ \\
\hline 0.005454 & $1\langle$ frac $/\rangle$ & 0.004903 & $\langle/ \sup \rangle+$ & 0.005077 & $y\langle\sup \rangle$ & 0.004792 & $=0$ & 0.005513 & 1) \\
\hline
\end{tabular}

Table 9. Most Popular Bigrams

\section{Kreyszig}

Freq (\%) Sequence $0.001049(x, y)$ $0.000951 y^{\langle\text {sup }\rangle} / /\langle$ sup $\rangle$ $0.000816 x^{\langle\mathrm{sub}\rangle} 1\langle/ \mathrm{sub}\rangle+$ $0.000812 f(x)=$ $0.000803 \int\langle$ sub $\rangle\langle\langle/$ sub $\rangle\langle$ sup $\rangle$ $0.0007280\langle/$ sub $\rangle\langle$ sup $\rangle \infty\langle/$ sup $\rangle$ $0.000722\langle/ \mathrm{sub}\rangle\langle\mathrm{sup}\rangle\langle/ \mathrm{sup}\rangle=0.000699(x, t)$ $0.000706\langle$ sup $\rangle / /\langle/$ sup $\rangle+$ $0.000635-z^{\langle\mathrm{sub}\rangle} 0\langle/ \mathrm{sub}\rangle$ $0.000599 \ldots$

0.000566 〈sub〉 1 〈/sub〉 〈sup〉। $\left.0.000553 z^{\langle\mathrm{sub}\rangle} 0\langle/ \mathrm{sub}\rangle\right)$ $0.000551 y^{\text {〈sub }} 1$ 〈/sub〉 〈sup〉 $0.000545 y(0)=$ $0.0005431\langle/ \mathrm{sub}\rangle\langle$ sup $\rangle\langle/$ sup $\rangle$ $0.000512\langle$ sub $\rangle\langle/ \mathrm{sub}\rangle\langle$ sup $\rangle$ $0.000508 z-z^{\langle\mathrm{sub}\rangle} 0$ $0.000501, y^{\langle\mathrm{sub}\rangle} 2\langle/ \mathrm{sub}\rangle$ $0.000497 y^{\langle\text {sub }\rangle} 2\langle/$ sub $\rangle\langle$ sup $\rangle$ $0.000495\langle$ sub $\rangle+1\langle/$ sub $\rangle$ $0.0004892^{\langle/ \mathrm{sub}\rangle\langle\text { sup }\rangle\langle/ \text { sup }\rangle}$ $0.000487 x^{\langle\mathrm{sub}\rangle} 2^{\langle/ \mathrm{sub}\rangle}=$ $0.000485 f(x, y$ $0.001295(x, y)$ $0.000494(x, y$
Greenberg

Freq $(\%)$ Sequence $0.001415 \int\langle\mathrm{sub}\rangle \mid\langle/ \mathrm{sub}\rangle\langle\mathrm{sup}\rangle \quad 0.001488 \int\langle\mathrm{sub}\rangle\langle\langle/ \mathrm{sub}\rangle\langle$ sup $\rangle$ $0.0007740\langle/$ sub $\rangle\langle$ sup $\rangle\left\langle\langle/\right.$ sup $\rangle 0.001001 \sum\langle$ sub $\rangle=1$

$0.000770 x^{\langle\text {sup }\rangle} 2^{\langle/ \mathrm{sup}\rangle}+\quad 0.000999\langle$ sub $n=1\langle/ \mathrm{sub}\rangle$ $0.000995 n=1\langle/$ sub $\rangle\langle$ sup $\rangle$ $0.0009351\langle/$ sub $\rangle\langle$ sup $\rangle \infty\langle/$ sup $\rangle$ $0.000927=1\langle/$ sub $\rangle\langle$ sup $\rangle \infty$ $0.000898) \sin ($ $0.000615 y(x)=$

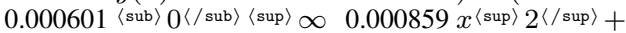
$0.000571\langle/ \mathrm{sub}\rangle(x)=$ $0.000566 i$ (sup $\rangle \wedge\langle/$ sup $\rangle+$ $0.000562(0)=0$ $0.000561 f(x, y$

$2\left\langle\right.$ sub〉 1 〈/sub〉 $(x \quad 0.000678-1)^{\langle\text {sup }} n$ $0.0005221</$ sub $\rangle(x)$ $0.0005151</$ sub $\rangle\langle$ sup $\rangle \infty\langle/$ sup $\rangle 0.000643-\infty\langle/$ sub $\rangle\langle$ sup $\rangle \infty$ $0.000513 x, y, z$ $0.000505 f(x)=$ $0.000444\langle$ sup $\rangle\langle/$ sup $\rangle\langle$ sub $\rangle \theta$ $0.000444 \Upsilon /$ sup $\rangle\langle$ sub $\rangle \theta^{\langle/ \text {sub }\rangle}$ $0.000438\langle$ sub $\rangle n^{\langle/ \mathrm{sub}\rangle}(x$ $0.000438 \operatorname{sinn} \pi$ $0.000839(-1)\langle$ sup

$0.000820 \sin (n$

$0.000721, \ldots$ $.000651(x, y$

$0.000643 \infty\langle/$ sub $\rangle\langle$ sup $\rangle \infty^{\langle/ \text {sup }\rangle}$ $0.000641 z\langle$ sub $\rangle\langle/$ sub $\rangle)$ $0.000620\langle$ sub $\rangle-\infty\langle/$ sub $\rangle\langle$ sup $\rangle$ $0.000620\langle/ \mathrm{sup}\rangle \sin ($ $0.000613 \int\langle\mathrm{sub}\rangle-\infty\langle/ \mathrm{sub}\rangle$ 0.000611 〈/sup〉 $\cos ($ $0.000599) \cos ($
Lopez

Freq (\%) Sequence 0.00284500000

$0.001361(x, y)$

$0.001202 x^{\langle\text {sup }\rangle} 2^{\langle/ \text {sup }\rangle}+$ $0.001045 \int\langle$ sub $\rangle\langle/$ sub $\rangle\langle$ sup $\rangle$ $0.000959 f(x)=$ $0.000736 x$ 〈sup $\rangle 2^{\langle/ \text {sup }\rangle}$

$0.000703(x, y$,

$0.000663\langle$ sup $\rangle 2^{\langle/ \text {sup }\rangle}+1$

$0.000647, \ldots$

$0.000644 f(x, y$

$0.000643+y^{\langle\text {sup }\rangle} 2^{\langle/ \text {sup }\rangle}$

$0.000609 x, y, z$

$0.000604, y, z)$

$0.0005882 x\langle$ sup $\rangle\langle/$ sup $\rangle$

$0.000585\langle$ sup $\rangle 2^{\langle/ \text {sup }\rangle}+y$

$0.000579) \sin ($

$0.000576\langle/$ sup $\rangle \cos ($

$0.000569\langle/ \mathrm{sup}\rangle \sin ($

$0.0005520\langle/ \mathrm{sub}\rangle\langle$ sup $\rangle \infty\langle/ \mathrm{sup}\rangle$

$0.0005162^{\langle/ \mathrm{sup}\rangle}+y^{\langle\sup \rangle}$

$0.000500 \sin (t$

$0.000499\langle/$ sup $\rangle+y^{\langle\text {sup }\rangle} 2$

$0.000478 \cos (t$

$0.000476\langle$ sub $\rangle\langle/$ sub $\rangle(x$
MSKit

Freq (\%) Sequence $0.004420 \mathrm{lim}^{\langle\mathrm{sub}\rangle} \mathrm{x}$ $0.004055 \mathrm{im}^{\langle\mathrm{sub}\rangle} x \rightarrow$ $0.003200 x$ 〈sup $\rangle 2^{\langle/ \text {sup }\rangle}+$ $0.002851 d y\langle$ frac $/\rangle d x$ $0.001996 f(x)=$ $0.001996 \sin (x$ $0.001901 x^{\langle\text {sup }\rangle} 2^{\langle/ \mathrm{sup}\rangle}-$ 0.001885 in $(x)$ $0.0015362 x^{\text {sup }\rangle} 2^{\langle/ \text {sup }\rangle}$ $0.001410 \cos (x$ 0.001330 os $(x)$ $0.001314 x^{\langle\text {sup }\rangle} 3\langle/$ sup $\rangle+$ $0.001235\langle$ sup $\rangle 2\langle/$ sup $\rangle+1$ $0.001219 \log \langle$ sub $\rangle$ $0.001219 o g^{\langle\text {sub }} a^{\langle/ \mathrm{sub}}$ $0.001172 y^{\langle\text {frac } /\rangle} d x=$ $0.001156<$ root $/>2 x^{\langle\text {sup }\rangle} 2$ $0.001156\left(x^{\langle\text {sup }\rangle} 2^{\langle/ \text {sup }}\right.$ $0.001156 d u^{\langle\mathrm{frac} /\rangle} d x$ $0.001156 g^{\langle\mathrm{sub}\rangle} a^{\langle/ \mathrm{sub}\rangle}($ $0.001093=\log ^{\langle\mathrm{sub}\rangle}$ $0.001093 \ln (x)$ 0.001013 〈sup $\rangle\langle/$ sup $\rangle(x$ $0.001013 y=f(x$

Table 10. Most Popular 5-grams 